\title{
Trauma to Tumor: A Hunt of Adenomatoid Odontogenic Tumor-A Rare Case Report
}

\author{
Ashish Katiyar ${ }^{1}$, Sukriti Gupta², Kirtija Gupta ${ }^{3}$, Mansi Pandey ${ }^{4}$
}

\begin{abstract}
Tumors and tumor-like growths arising from odontogenic tissues constitute a heterogeneous group of interesting lesions, as they display the various inductive interactions that normally occur among the embryologic components of the developing tooth germ. Adenomatoid odontogenic tumor is a benign tumor, most common in the maxilla, characterized by slow, progressive, painless growth of firm consistency, the size of which usually does not reach large proportions. They are of three types: extrafollicular, follicular, and peripheral. It usually develops around or overlaps adjacent teeth.

Keywords: Enucleation, Hamartoma, Odontogenic tumor, Trauma.

International Journal of Clinical Pediatric Dentistry (2019): 10.5005/jp-journals-10005-1655
\end{abstract}

\section{INTRODUCTION}

Adenomatoid odontogenic tumor is a benign, hamartomatous, noninvasive, uncommon, and epithelial lesion of the odontogenic origin. ${ }^{1}$ It is a benign lesion from the dental lamina or its remains. It is a relatively uncommon neoplasm that accounts for about $3 \%$ of all odontogenic tumors being more common in the maxilla; in females, it is more often associated with impacted canines. Previously it was known as pseudo-adenoameloblastoma by Dreibladt; later, Staphne first recognized this as a distinct pathological entity. ${ }^{2,3}$

The idea of origin of this lesion from reduced enamel epithelium is enforced by many ultrastructural and immunohistochemical studies along with the resemblance of cytological features seen in this lesion to components derived from the enamel organ; the occurrence in tooth-bearing region of jaws and its unavoidable alliance with impacted teeth has further strengthened this notion. ${ }^{4}$ The 1971 WHO classification stated: "It is generally believed that the lesion is not a neoplasm." However, in 2005, WHO defined AOT as a tumor composed of the odontogenic epithelium, presenting a variety of histoarchitectural patterns, embedded in mature connective tissue stroma, and characterized by a slow and progressive growth. ${ }^{5}$ Radiographically, these lesions are similar to other lesions of odontogenic origin. Nevertheless, these characteristics depend on the tumor type. In the follicular type, they appear as a well-defined and circumscribed area that is associated to the crown or root of an impacted tooth. In the extrafollicular type, the observed lesion is located between or over the roots of an erupted tooth. ${ }^{6}$ In the peripheral type, thinning or resorption of the cortical cortex can be observed, while in some cases, tooth displacement without root resorption is noted. Nevertheless, in periapical lesions, radiopacities have been observed within the lesion up to $78 \%$, which represents an advantage compared to orthopantomography. ${ }^{7}$

\section{Case Description}

A 13-year-old female patient was reported to the Department of Pedodontics and Pediatric Dentistry with the chief complaint of an unerupted front tooth (Fig. 1). The patient had the history of trauma 7 years back and no treatment was taken. Extraorally, the face was

\begin{abstract}
1,3 Department of Pedodontics and Preventive Dentistry, Faculty of Dental Sciences, Rama Dental College, Rama University, Kanpur, Uttar Pradesh, India

${ }^{2}$ Department of Pedodontics and Preventive Dentistry, Maharana Pratap Dental College, Kanpur, Uttar Pradesh, India

${ }^{4}$ Department of Pedodontics and Preventive Dentistry, Rama Dental College Hospital and Research Centre, Kanpur, Uttar Pradesh, India

Corresponding Author: Ashish Katiyar, Department of Pedodontics and Preventive Dentistry, Faculty of Dental Sciences, Rama Dental College, Rama University, Kanpur, Uttar Pradesh, India, Phone: +91 5122583785, e-mail: drashish1981@gmail.com
\end{abstract}

How to cite this article: Katiyar A, Gupta S, et al. Trauma to Tumor: A Hunt of Adenomatoid Odontogenic Tumor-A Rare Case Report. Int J Clin Pediatr Dent 2019;12(4):366-369.

Source of support: Nil

Conflict of interest: None

symmetric with no swelling on her face. Intraorally, missing 21 was noticed with a small bulge on the anterior maxillary left front teeth region extending from mesial aspect of 11 to distal aspect of 22, roughly oval in shape and measuring about $1 \mathrm{~cm} \times 2 \mathrm{~cm}$ in the greatest dimension. The swelling was hard in consistency and nontender on palpation. The radiographical analysis showed the presence of unerupted permanent left central incisor with the underlying pathology (Fig. 2). On the basis of the history and clinical examination, a provisional diagnosis of the dentigerous cyst was given. The underline pathology was surgically enucleated with the removal of the unerupted central incisor and was sent to the histopathological examination, which revealed it as an AOT (Figs 3 to 6).

The recurrence rate for AOT is rare and the prognosis is good. The present case has been on follow-up since 12 months after the surgery; no recurrence is noted (Figs 7 to 10 ).

\section{Discussion}

AOT is also called "two-thirds tumor" because two-thirds occur in young females, two-thirds of these cases occur in the maxilla, twothirds of these tumors are associated with unerupted teeth, and two-thirds occur in canines. The lesions are usually asymptomatic 


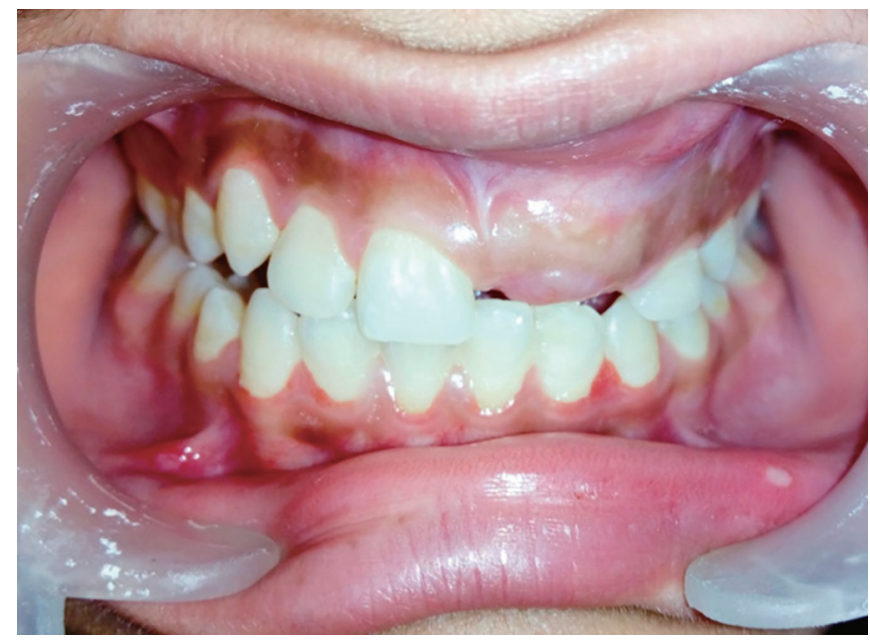

Fig. 1: Clinical preoperative (intraoral)

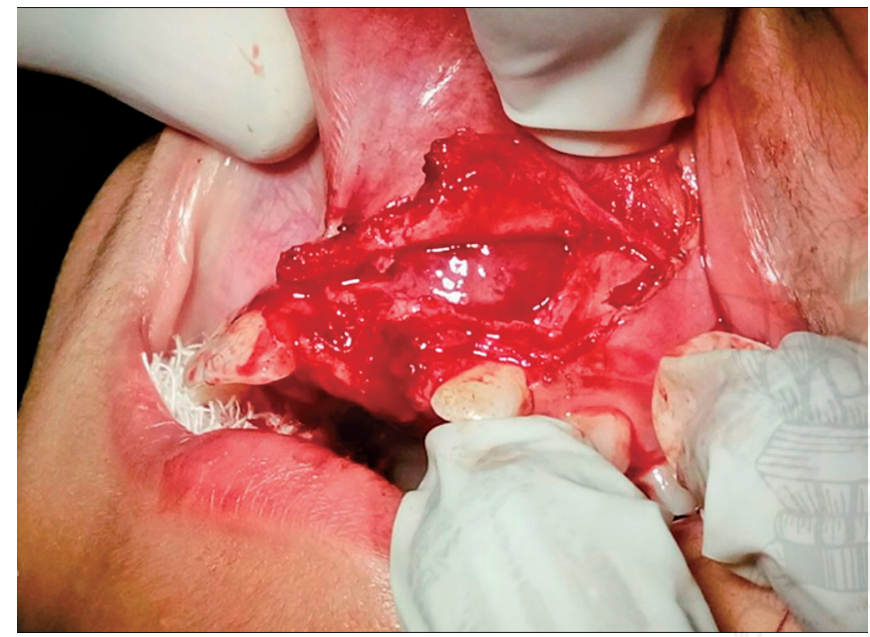

Fig. 3: Mucoperiosteal flap elevated

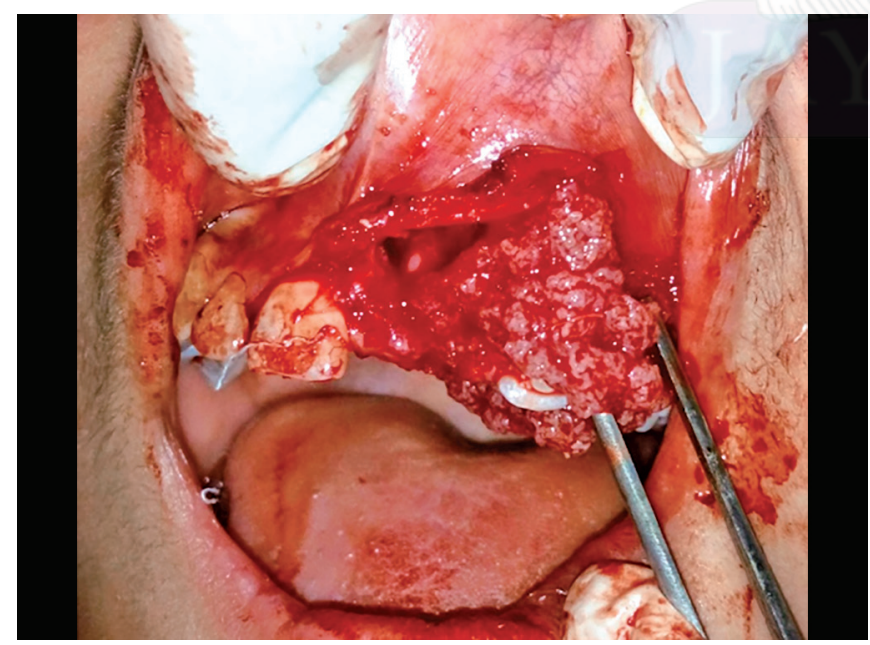

Fig. 5: Unerupted left central incisor was completely covered with pathology

and is often associated with cortical expansion. The involved teeth are commonly impacted and adjacent teeth may be slightly displaced. All the above-mentioned features were concurrent with

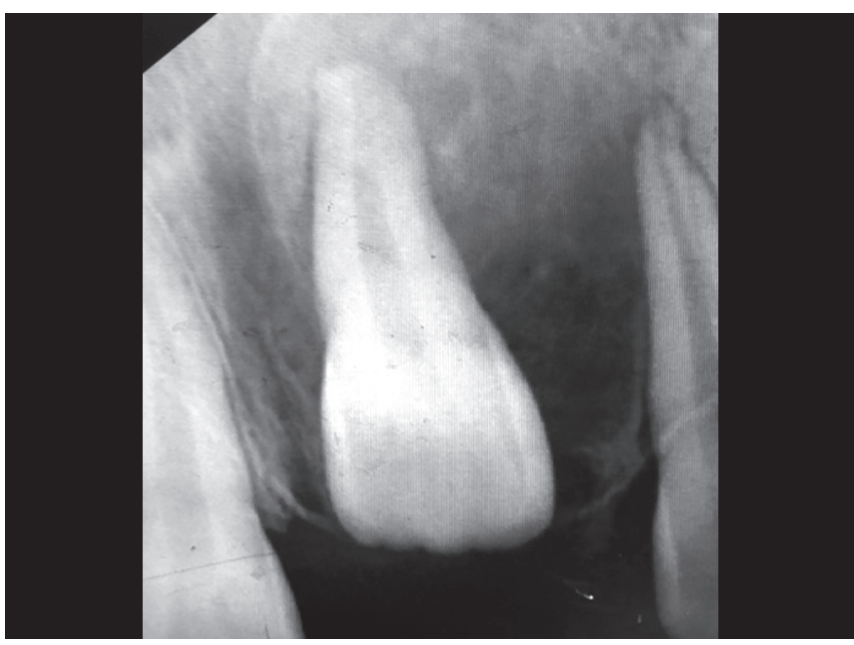

Fig. 2: Preoperative radiographic

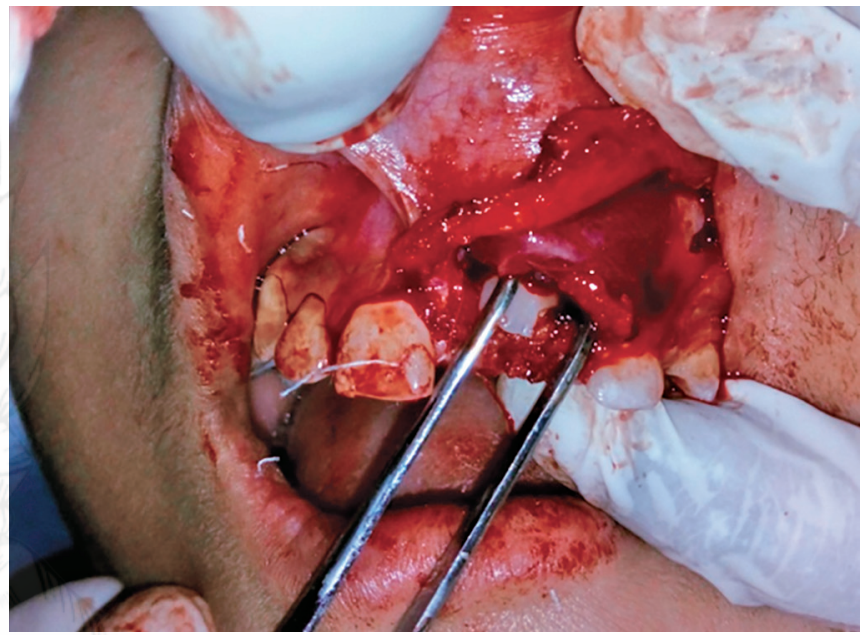

Fig. 4: Unerupted left central incisor is visible

our case (Fig. 7). ${ }^{8}$ In general, the tumor does not exceed $1-3 \mathrm{~cm}$ in its greatest diameter and usually occurs within the tooth-bearing areas of jaws and often associated with impacted teeth (Fig. 8). ${ }^{9}$

Peripheral crown radio-lucid images found in upper or lower jaws are more common than they appear. AOT exhibits a radiolucid image that is limited to the non-erupted tooth enamel-dentin junction, at the anterior segment of the upper jaw. Not withstanding the fact that rare cases of this disease have been reported in the lower jaw, canines are the teeth most affected by this condition (Fig. 9). ${ }^{10,11}$

It is composed of spindle-shaped or polygonal cells forming sheets and whorled masses in a scarce connective tissue stroma. The characteristic duct-like structures are lined by a single row of columnar epithelial cells and the nuclei are polarized away from the central lumen. The lumen may contain amorphous eosinophilic material or it may be empty as seen in our case (Fig. 10). ${ }^{12}$

Histologically, this tumor is well defined and refers to the presence of various arteries, from the odontogenic epithelium, grouped in structures similar to rosettes and scrotal connective tissue stroma, the presence of follicular structures with palisade and polarized cells, and regions with presence of area in calcification. It is a capsule of dense connective tissue, proliferation of tumor cells forming ductal structures and follicular structures with cylindrical 


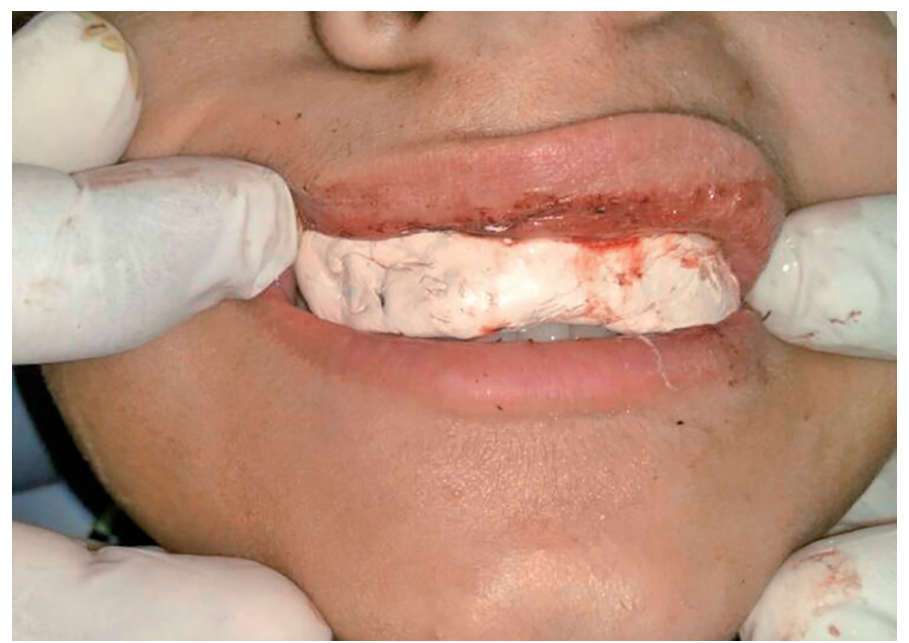

Fig. 6: Periodontal dressing was given

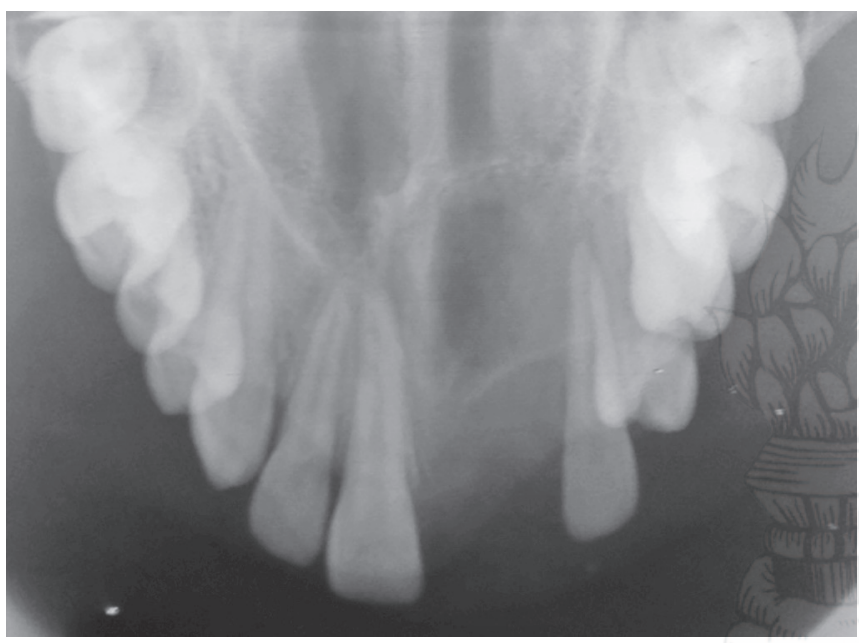

Fig. 7: Postsurgical maxillary occlusal view (3 months)

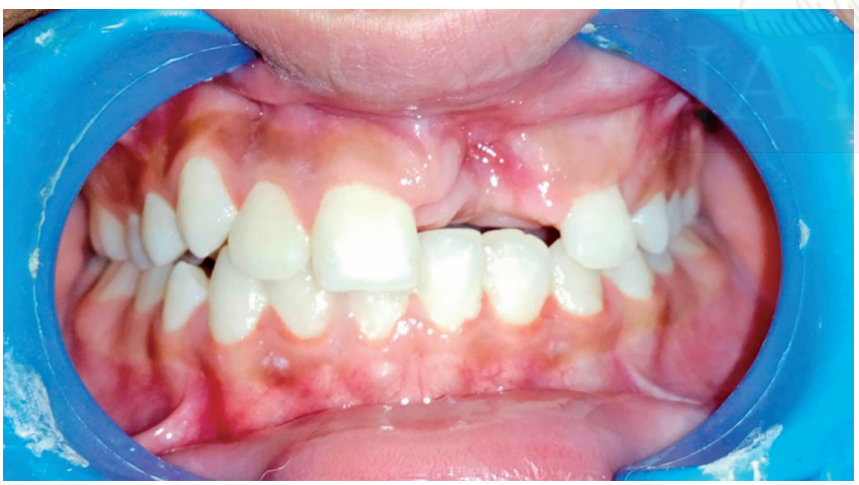

Fig. 9: Postsurgical healing

cells in palisade and polarized nuclei, and the presence of foci of calcification. The histological finding of the piece corroborates the majority of the authors. . $^{13,14}$

\section{Conclusion}

From above case report, we interpret that trauma can turn into an odontogenic lesion if not taken care at the initial stages of life; however, the recurrence rate is not high. The treatment of choice for

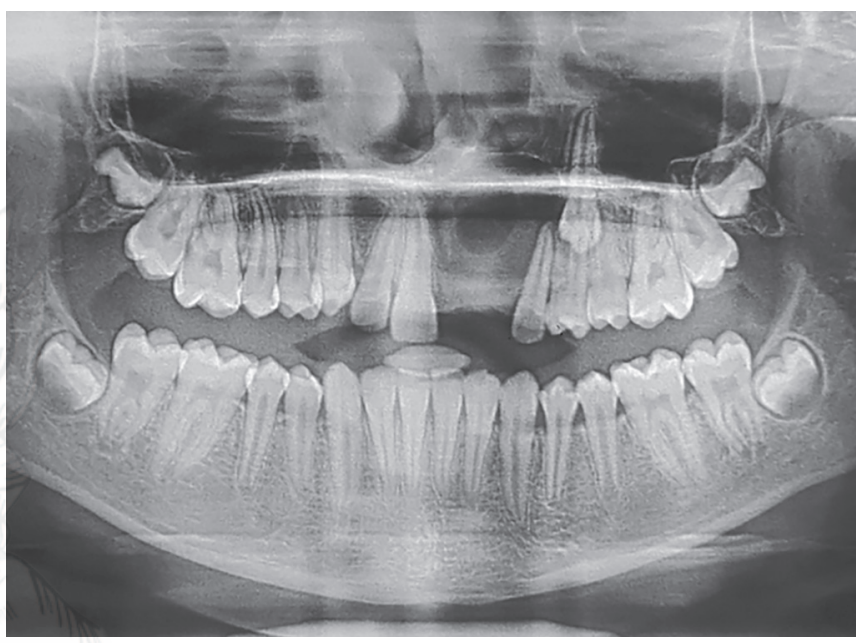

Fig. 8: OPG after 6 months

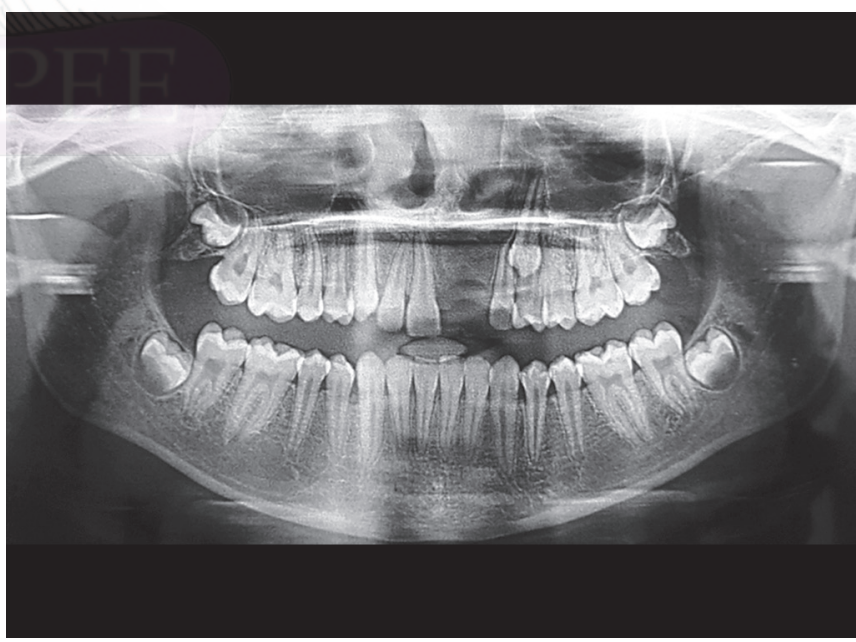

Fig. 10: OPG after 12 months

AOT is surgical with enucleation. The tumor does not usually have aggressive behavior, and recurrences are rare. Early diagnosis of this by the dental surgeon is mandatory when a clinical sign is mentioned, and early enucleation prevents an excessive destruction of bone. 


\section{References}

1. Prakasam M, Tiwari S, et al. Adenomatoid odontogenic tumour. BMJ Case Rep 2013 Jun;2013:bcr2013010212. DOI: 10.1136/bcr-2013010212.

2. Philipsen HP, Samman N, et al. Variants of the adenomatoid odontogenic tumor with a note on tumor origin. J Oral Pathol Med 1992;21:348-352. DOI: 10.1111/j.1600-0714.1992.tb01363.x.

3. Reichart PA, Philipsen HP. Odontogenic Tumors and Allied Lesions. United Kingdom: Quintessence Publishing Co. Ltd; 2004; pp. 105-115.

4. Kaminagakura E, Costa MS, et al. Extrafollicular adenomatoid odontogenic tumor: A case report. Gen Dent 2007;55:141-142.

5. Motamedi $\mathrm{MH}$, Shafeie $\mathrm{HA}$, et al. Salvage of an impacted canine associated with an adenomatoid odontogenic tumour: A case report. Br Dent J 2005;199:89-90. DOI: 10.1038/sj.bdj.4812522.

6. Jham BC, Passos JB, et al. Adenomatoid odontogenic tumor originated in the periodontal ligament. Oral Oncol Extra 2006;42:268-271. DOI: 10.1016/j.ooe.2006.05.003.

7. Vera Sempere FJ, Artes Martínez MJ, et al. Follicular adenomatoid odontogenic tumor: Immunohistochemical study. Med Oral Patol Oral Cir Bucal 2006;11:E305-E308.
8. Nigam S, Gupta SK, et al. Adenomatoid odontogenic tumor - A rare cause of jaw swelling. Braz Dent J 2005;16:251-253. DOI: 10.1590/ S0103-64402005000300015.

9. Effiom OA, Odukoya O. Adenomatoid odontogenic tumour: A clinicopathological analysis and melanin pigmentation study of 31 Nigerian cases. Niger Postgrad Med J 2005;12:131-135.

10. Bravo $M$, White $D$, et al. Adenomatoid odontogenic tumor mimicking a dentigerous cyst. Int J Pediatr Otorhinolaryngol 2005;69:1685-1688. DOI: 10.1016/j.ijporl.2005.03.055.

11. Handschel JG, Depprich RA, et al. Adenomatoid odontogenic tumor of the mandible: Review of the literature and report of a rare case. Head Face Med 2005;1:3. DOI: 10.1186/1746-160X-1-3.

12. Carlos-Bregni R, Vargas PA, et al. Adenomatoid odontogenic hamartoma: Concerns about correct nomenclature and 2 additional case reports. J Oral Maxillofac Surg 2009;67:1779-1780. DOI: 10.1016/ j.joms.2008.12.062.

13. Ali YH, Hussain AE. Adenomatoid odontogenic tumour of the middle turbinate: Case report and literature review. J Otolaryngol Head Neck Surg 2009;38:E9-E13.

14. Yilmaz N, Acikgoz A, et al. Extrafollicular adenomatoid odontogenic tumor of the mandible: Report of a case. Eur J Dent 2009;3:71-74. 